\title{
Irreversible Electroporation to Treat Malignant Tumor Recurrences Within the Pelvic Cavity: A Case Series
}

\author{
L. G. P. H. Vroomen ${ }^{1}\left(\mathbb{D} \cdot\right.$ H. J. Scheffer ${ }^{1}$ - M. C. A. M. Melenhorst ${ }^{1}$ • \\ N. van Grieken ${ }^{2}$ M. P. van den Tol $^{3} \cdot$ M. R. Meijerink ${ }^{1}$
}

Received: 17 December 2016/ Accepted: 19 April 2017/Published online: 3 May 2017

(c) The Author(s) 2017. This article is an open access publication

\begin{abstract}
Objective To describe the initial experience with irreversible electroporation (IRE) to treat pelvic tumor recurrences.

Methods A retrospective single-center analysis was performed. Adverse events were recorded using Common Terminology Criteria of Adverse Events (CTCAE) 4.0. Clinical outcome was determined using pain- and generalsymptom assessment, including Seddon's peripheral nerve injury (PNI) types. Radiological outcome was evaluated by comparing baseline with three-month 18F-FDG PET-CT follow-up.

Results Eight patients (nine tumors [recurrences of primary rectal $(n=4)$, anal $(n=1)$, sigmoid $(n=1)$, cervical $(n=1)$, and renal cell carcinoma $(n=1)])$ underwent percutaneous IRE as salvage therapy. Median longest tumor diameter was $3.7 \mathrm{~cm}$ (range 1.2-7.0). One CTCAE grade III adverse event (hemorrhage) and eight CTCAE grade II complications occurred in 6/8 patients: vagino-tumoral fistula $(n=1)$, lower limb motor loss ( $n=3$; PNI type II) with partial recovery in one patient, hypotonic bladder ( $n=2$; PNI types I and II) with complete recovery in one patient, and upper limb motor loss
\end{abstract}

L. G. P. H. Vroomen

la.vroomen@vumc.nl

1 Department of Radiology and Nuclear Medicine, VU University Medical Center, de Boelelaan 1117, 1081 HV Amsterdam, The Netherlands

2 Department of Pathology, VU University Medical Center, de Boelelaan 1117, 1081 HV Amsterdam, The Netherlands

3 Department of Surgical Oncology, VU University Medical Center, de Boelelaan 1117, 1081 HV Amsterdam, The Netherlands
( $n=2$; PNI type II) with partial recovery in both patients. No residual tumor tissue was observed at 3-month followup. After a median follow-up of 12 months, local progression was observed in $5 / 9$ lesions $(4 / 5$ were $>3 \mathrm{~cm}$ preIRE); one lesion was successfully retreated. Debilitating preprocedural pain $(n=3)$ remained unchanged $(n=1)$ or improved $(n=2)$.

Conclusion IRE may represent a suitable technique to treat pelvic tumor recurrences, although permanent neural function loss can occur. Complete ablation seems realistic for smaller lesions; for larger lesions symptom control should be the focus.

Keywords Ablation · Feasibility · Locoregional neoplasm recurrence $\cdot$ Pelvic region - Peripheral nerves
Abbreviations
ASA
$\mathrm{Ce}$
American Society of Anesthesiologists
CT
Contrast-enhanced
CTCAE
Computed tomography
18F-FDG PET 18F-fluorodeoxyglucose positron emission tomography
IONM
Intraoperative neurophysiologic
IRE
LSR monitoring
PNI
RECIST
Irreversible electroporation
Local site recurrence
Peripheral nerve injury
RFA
SABR 
TDP

PERCIST

TLP

VAS
Time to distant progression

PET Response Criteria in Solid Tumors

Time to local progression

Visual Analog Scale

\section{Introduction}

Present local tumor recurrences are a challenge for any malignancy in any location. Tumors that recur in the pelvis may be even more challenging to treat. Malignancies that are notorious for their recurrence within the pelvis following radiotherapy and/or surgery are both female and male urogenital tract tumors and locoregional recurrences from gastrointestinal origin such as anorectal carcinomas $[1,2]$. Due to ingrowth in or compression on peripheral nerves, these relapsing malignancies can cause aggravating pain and neural function loss. Many of these patients have undergone multiple prior resections resulting in scar and adhesions, most have undergone some form of systemic therapy, and many have received external beam radiation. The presence of extensive adhesions induced by previous surgical procedures and the risk of radiation-induced toxicity in a previously irradiated area precludes radical local treatment options such as repeat surgery [3] and stereotactic ablative body radiation therapy (SABR) [4, 5]. The risk of severe treatment-induced morbidity does not seem to outweigh clinical benefit $[2,6]$. In general, therapy for this specific patient population primarily aims at prolonging thepreferably quality preserved-life span, and most patients will be referred to medical oncologists for either palliative chemotherapy or the best supportive care [7,8].

Yet, selected patients can be offered other local treatment modalities such as radiofrequency ablation (RFA) or cryotherapy [9]. One important drawback of these thermal treatment modalities is the high risk of inducing thermal damage to important neural structures like the sciatic nerve or perisacral plexus, as well as to the intestines, bladder, ureters, and large pelvic vessels [10].

Irreversible electroporation (IRE) is an emerging ablation technique that is based on the application of an electric field across cells that alters the transmembrane potential. On reaching a sufficiently high voltage, the phospholipid bilayer structure of the cell membrane is permanently disrupted, inducing apoptosis [11, 12]. Electroporation is regarded to leave supporting tissue largely unaffected, so the structural integrity of large blood vessels and intestines is relatively preserved $[11,13]$. Moreover, initially damaged axons may regenerate with complete recovery of function, according to

preclinical animal studies [14-17]. For these reasons, IRE may prove a safe and feasible treatment option for patients with malignant tumor recurrences within the pelvis that are considered unsuitable for established focal therapies [14, 15, 17].

This retrospective study describes the preliminary single-center experience of eight patients (nine lesions) with locoregional malignant tumor recurrences within the pelvis that were treated with percutaneous IRE in terms of morbidity and disease control.

\section{Materials and Methods}

A retrospective analysis was performed of all patients treated with IRE for malignant pelvic tumor recurrences that were considered unsuitable for additional resection, re-radiation, or thermal ablation techniques due to the vicinity of major nerves, prostate, ureter, or intestines. Further systemic chemotherapy was considered unfavorable. All patients were discussed in our weekly multidisciplinary oncology board, which consisted of at least a medical oncologist, radiation therapist, surgical oncologist, diagnostic abdominal and interventional radiologist, and pathologist. IRE was only performed if the oncology board unanimously agreed on the indication. Treatment with IRE was considered off-label use within the scope of an individual treatment decision. Patients were considered appropriate IRE candidates if they met the following criteria: (1) IRE was used as salvage therapy, (2) a reasonable functional reserve [American Society of Anesthesiologists (ASA) performance status $\leq 3$ ], and (3) the absence of ventricular arrhythmias. Histopathological proof of recurring malignancy was required, and the lesions had to be proven 18F-fluorodeoxyglucose positron emission tomography (18F-FDG PET) avid prior to IRE. A history of epilepsy was considered an exclusion criteria. Local review board approval for the study was obtained, and all patients provided written informed consent. The study was conducted conformal to the guidelines for Good Clinical Practice and the Declaration of Helsinki.

\section{IRE Procedure}

All procedures were performed under general anesthesia with propofol, sufentanil, and rocuronium and maintained with propofol and remifentanil. To define the three-dimensional measurements of the tumor and its vicinity to vital structures, a contrast-enhanced computed tomography (ce-CT) scan was performed, using multiplanar image reconstruction. The size and shape of the tumor, including 
a 5-mm tumor-free margin, determined the number and configuration of the needle electrodes. Needle electrodes with an exposure length of $15 \mathrm{~mm}$ were percutaneously positioned in and around the tumor under CT fluoroscopy guidance, aiming at an interneedle distance of 15-20 mm. The NanoKnife (AngioDynamics Inc, Latham, NY) was used to perform the procedure. A total of 100 pulses of $1500 \mathrm{~V} / \mathrm{cm}$ with a $90-\mu$ s pulse length were delivered for each electrode pair. For larger tumors, the electrodes were pulled back $1.0 \mathrm{~cm}$ to ablate the superficial part of the tumor.

\section{Safety Assessment}

Immediately after the procedure, a second ce-CT scan was acquired to assess the ablation zone and to detect early complications such as perilesional bleeding. All direct and indirect procedure-related complications were scored according to the Common Terminology Criteria of Adverse Events (CTCAE), version 4.0. Pain assessment was determined using the Visual Analog Scale (VAS) scores prior to IRE, and $24 \mathrm{~h}$ and 3 months after the procedure. Physical examinations were performed pre- as well as post-procedurally in order to identify IRE-induced nerve loss. Neurological impairment was evaluated using Seddon's classification [18]. Based on the severity of the peripheral nerve injury (PNI), the prognosis, and the recovery time, three types of neural damage-neurapraxia, axonotmesis, and neurotmesis-are defined within the Seddon's classification [18]. Neurapraxia describes the mildest type and refers to a block to conduction of nerve impulses but without interruption of the axon or perineurium [19]. Axonotmesis is the second type and refers to an injured axon, yet the surrounding connective tissue remains intact [19]. Neurotmesis is considered the most severe injury type in which the axon as well as the connective framework is damaged [19] (Table 1).

\section{Oncological Outcome}

Outcome was evaluated comparing baseline with threemonth 18F-FDG PET-CT, using both the revised Response Evaluation Criteria In Solid Tumors (RECIST) [20] and the PET Response Criteria In Solid Tumors (PERCIST) [21] to obtain primary and assisted efficacy rates, time to local progression (TLP), and time to distant progression (TDP). Imaging using PET-CT was chosen because of its ability to detect tumor residue or progression in an area that is characterized by a disturbed anatomy. Since IRE induces local inflammation post-IRE, three-month follow-up was preferred above monitoring at an earlier time point. Based on imaging features, the parameters for local site recurrence (LSR) were (a) tumor lesion increase of at least $20 \%$ in longest diameter compared to the baseline scan at 3 months post-IRE, or (b) a more than $30 \%$ increase in standardized uptake value corrected for lead body mass (SUL) peak or growth in lesion total lesion glycolysis by more than $75 \%$, without signs indicative for inflammation or abscess formation. Primary efficacy rate was defined as the percentage of lesions without signs for LSR at least 3 months after the initial IRE procedure, according to the above-mentioned criteria. Assisted efficacy rate was defined as the percentage of tumors completely eradicated at least 3 months after the last procedure, including tumors that underwent repeat ablation(s) [22].

\section{Results}

Between December 2012 and April 2016, eight patients (nine tumors) underwent percutaneous CT-guided IRE to treat recurrences of primary rectal $(n=4)$, anal $(n=1)$, sigmoid $(n=1)$, cervical $(n=1)$, and renal cell carcinoma $(n=1)$. Needle insertion and pulse delivery were successful in all procedures. Median longest tumor diameter was $3.7 \mathrm{~cm}$ (range 1.2-7.0). Patient and tumor characteristics are displayed in Table 2.

Table 1 Seddon's classification

\begin{tabular}{|c|c|c|c|c|}
\hline & Score & Tissue injured & Clinical findings & Prognosis \\
\hline Neurapraxia & I & Myelin & $\begin{array}{l}\text { Profound motor loss, paralysis } \\
\text { lasting days-months } \\
\text { Normal to minimal sensory } \\
\text { involvement }\end{array}$ & Excellent \\
\hline Axonotmesis & II & Myelin, axon & $\begin{array}{l}\text { Complete motor loss with } \\
\text { sensory involvement } \\
\text { OR } \\
\text { Complete motor loss with } \\
\text { normal sensation }\end{array}$ & Fair \\
\hline Neurotmesis & III & $\begin{array}{l}\text { Connective sheath damage ranges from partial disruption of the } \\
\text { endoneurium to complete disruption of the involved nerve }\end{array}$ & $\begin{array}{l}\text { Complete motor loss } \\
\text { Complete sensation loss }\end{array}$ & Poor \\
\hline
\end{tabular}




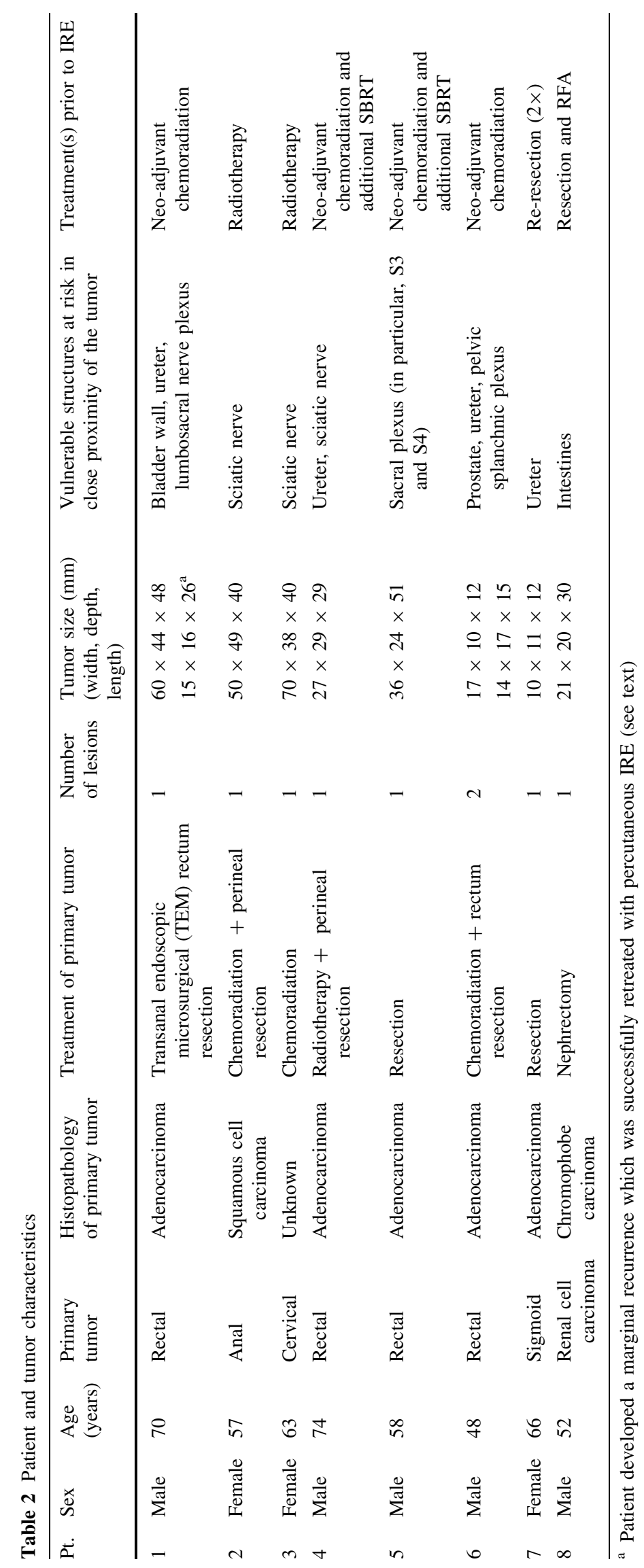




\section{Complications}

There were no deaths within 90 days post-IRE. One patient experienced a delayed hemorrhage after restarting anticoagulation therapy 3 days after the procedure (CTCAE grade III). Eight CTCAE grade II complications occurred in $6 / 8$ patients. Three patients showed lower limb motor loss [all PNI type II (axonotmesis)], with partial recovery in one patient. Two patients developed a hypotonic bladder [PNI type I (neurapraxia), and PNI type II (axonotmesis)] with complete recovery in one patient. Two patients showed upper limb motor loss, with partial recovery in both patients [PNI type II (axonotmesis)]. One patient developed a vagino-tumoral fistula following the IRE procedure. Procedure-related details including complications are summarized in Table 3.

\section{Follow-up}

In all patients, no residual tumor tissue was observed at 3 -month follow-up imaging. After a median follow-up of

12 months (range 4-36), four patients were still alive and four had deceased, respectively, 11, 12, 21, and 36 months after IRE. Although no LSR's have been objectified according to conventional RECIST so far, unequivocal LSR was observed in five patients (five lesions) using PERCIST criteria. For lesions with a largest tumor diameter of $\leq 3 \mathrm{~cm}(5 / 9)$, up until now, one LSR has been detected $(29 \mathrm{~mm})$. An example of a successfully treated lesion with a largest tumor diameter of $\leq 3 \mathrm{~cm}$ is shown in Fig. 1. Contrarily, all (4/9) lesions with a largest diameter of $>3 \mathrm{~cm}$ recurred. CT-guided core biopsy confirmed tumor relapse in one patient who was successfully retreated with percutaneous IRE 4 months after the initial treatment; hereafter, no local recurrence was detected until his death (patient 1, Fig. 2) (patient died of brain metastases). Tumor recurrence distant from the pelvic treatment site developed in 4/9 patients: cerebral $(n=1)$, pulmonary $(n=2)$, and in a different site in the pelvis $(n=1)$. Lesion-based primary efficacy rate was $33 \%$ (3/9), and assisted efficacy rate was $44 \%$ (4/9). Adjusted for tumor size, the primary efficacy was $80(4 / 5)$ and $0 \%(0 / 4)$ for tumors $\leq 3$ and $>3 \mathrm{~cm}$, respectively. Assisted efficacy was 80 (4/5) and 25\% (1/4), respectively. One day post-IRE, the reported pain was

Table 3 Procedure details, clinical and radiological outcome

\begin{tabular}{|c|c|c|c|c|c|c|c|c|c|c|}
\hline \multirow[t]{2}{*}{ Pt. } & \multirow[t]{2}{*}{$\begin{array}{l}\# \\
\text { Probes }\end{array}$} & \multirow[t]{2}{*}{$\begin{array}{l}\# \\
\text { Pullbacks }\end{array}$} & \multirow[t]{2}{*}{$\begin{array}{l}\text { Complications } \\
\text { (CTCAE } \\
\text { grade) }\end{array}$} & \multirow[t]{2}{*}{ Complication characteristics } & \multirow[t]{2}{*}{$\begin{array}{l}\text { Seddon's } \\
\text { classification }\end{array}$} & \multirow[t]{2}{*}{$\begin{array}{l}\text { Affected } \\
\text { nerve(s) }\end{array}$} & \multirow[t]{2}{*}{$\begin{array}{l}\text { Recovery } \\
\text { neural } \\
\text { function }\end{array}$} & \multirow[t]{2}{*}{$\begin{array}{l}\text { Follow- } \\
\text { up } \\
\text { (months) }\end{array}$} & \multicolumn{2}{|c|}{$\begin{array}{l}\text { Time to } \\
\text { progression } \\
\text { (months) }\end{array}$} \\
\hline & & & & & & & & & TLP & TDP \\
\hline 1 & 5 & 1 & - & - & - & - & - & $36^{\dagger}$ & $4 /-*$ & 3 \\
\hline 2 & 6 & 2 & II & $\begin{array}{l}\text { Lower limb motor } \\
\text { loss }+ \text { sensory involvement } \\
\text { Vagino-tumoral fistula }\end{array}$ & Axonotmesis & $\begin{array}{l}\text { Sciatic } \\
\text { nerve }\end{array}$ & Partial & $11^{\dagger}$ & 5 & - \\
\hline 3 & 6 & 2 & $\begin{array}{l}\text { II } \\
\text { II }\end{array}$ & $\begin{array}{l}\text { Hypotonic bladder } \\
\text { Lower limb motor } \\
\text { loss }+ \text { sensory involvement }\end{array}$ & $\begin{array}{l}\text { Neurapraxia } \\
\text { Axonotmesis }\end{array}$ & $\begin{array}{c}\text { Pudendal } \\
\text { plexus } \\
\text { S2-S4 } \\
\text { Sciatic } \\
\text { nerve }\end{array}$ & $\begin{array}{l}\text { Completely } \\
\text { None }\end{array}$ & $21^{\dagger}$ & 5 & 5 \\
\hline 4 & 4 & 1 & $\begin{array}{l}\text { II } \\
\text { III }\end{array}$ & $\begin{array}{l}\text { Slight deterioration of } \\
\text { preexisting lower limb motor } \\
\text { loss }+ \text { sensory involvement } \\
\text { Hemorrhage }\end{array}$ & Axonotmesis & $\begin{array}{l}\text { Sciatic } \\
\text { nerve }\end{array}$ & None & $12^{\dagger}$ & 6 & 6 \\
\hline 5 & 6 & 1 & II & Hypotonic bladder & Axonotmesis & $\begin{array}{l}\text { Pudendal } \\
\text { plexus } \\
\text { S2-S4 }\end{array}$ & None & 17 & 7 & - \\
\hline 6 & $\begin{array}{l}4 \\
4\end{array}$ & $\begin{array}{l}1 \\
0\end{array}$ & - & - & - & - & - & 9 & - & 9 \\
\hline 7 & 3 & 0 & II & $\begin{array}{l}\text { Upper limb motor } \\
\text { loss }+ \text { sensory involvement }\end{array}$ & Axonotmesis & $\begin{array}{c}\text { Femoral } \\
\text { nerve }\end{array}$ & Partial & 9 & - & - \\
\hline 8 & 6 & 0 & II & $\begin{array}{l}\text { Upper limb motor } \\
\text { loss }+ \text { sensory involvement }\end{array}$ & Axonotmesis & $\begin{array}{c}\text { Femoral } \\
\text { nerve }\end{array}$ & Partial & 4 & - & - \\
\hline
\end{tabular}

TLP time to local progression, TDP time to distant progression

* Patient developed a marginal recurrence which was successfully retreated with percutaneous IRE (see text)

$\dagger$ Deceased 
Fig. 1 18F-FDG PET-CT

image of a 48-year-old male patient with two small pathologically proven locoregional recurrences (arrows) of primary rectal adenocarcinoma in the precoccygeal and right periprostatic area (A). Nonenhanced CT scan showing the inserted needle electrodes prior to pulse delivery (B). 18F-FDG PET-CT image 3 months after IRE showing no signs for residual or recurring disease $(\mathbf{C})$
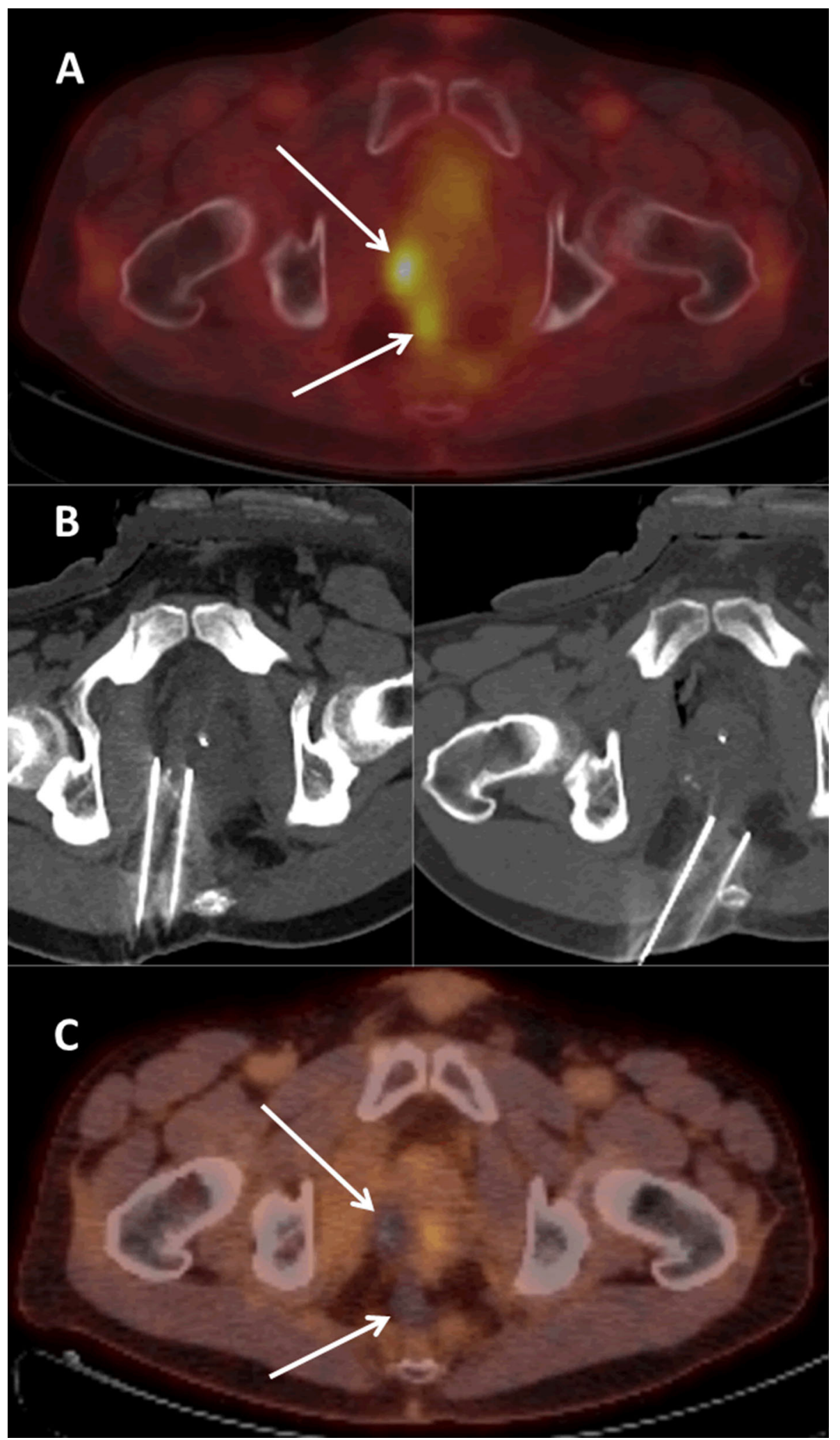

moderate with a median VAS score of 3 (range 0-5); pain could easily be controlled with acetaminophen combined with NSAIDs and opioids if needed. Prior to IRE, three patients reported debilitating pain; 3 months after IRE pain perception had remained unchanged in one patient (VAS score 5; patient 2) and had improved slightly (VAS score from 5 to 4; patient 5) and considerably (VAS score from 6 to 3 ; patient 3 ) in the other two patients. 

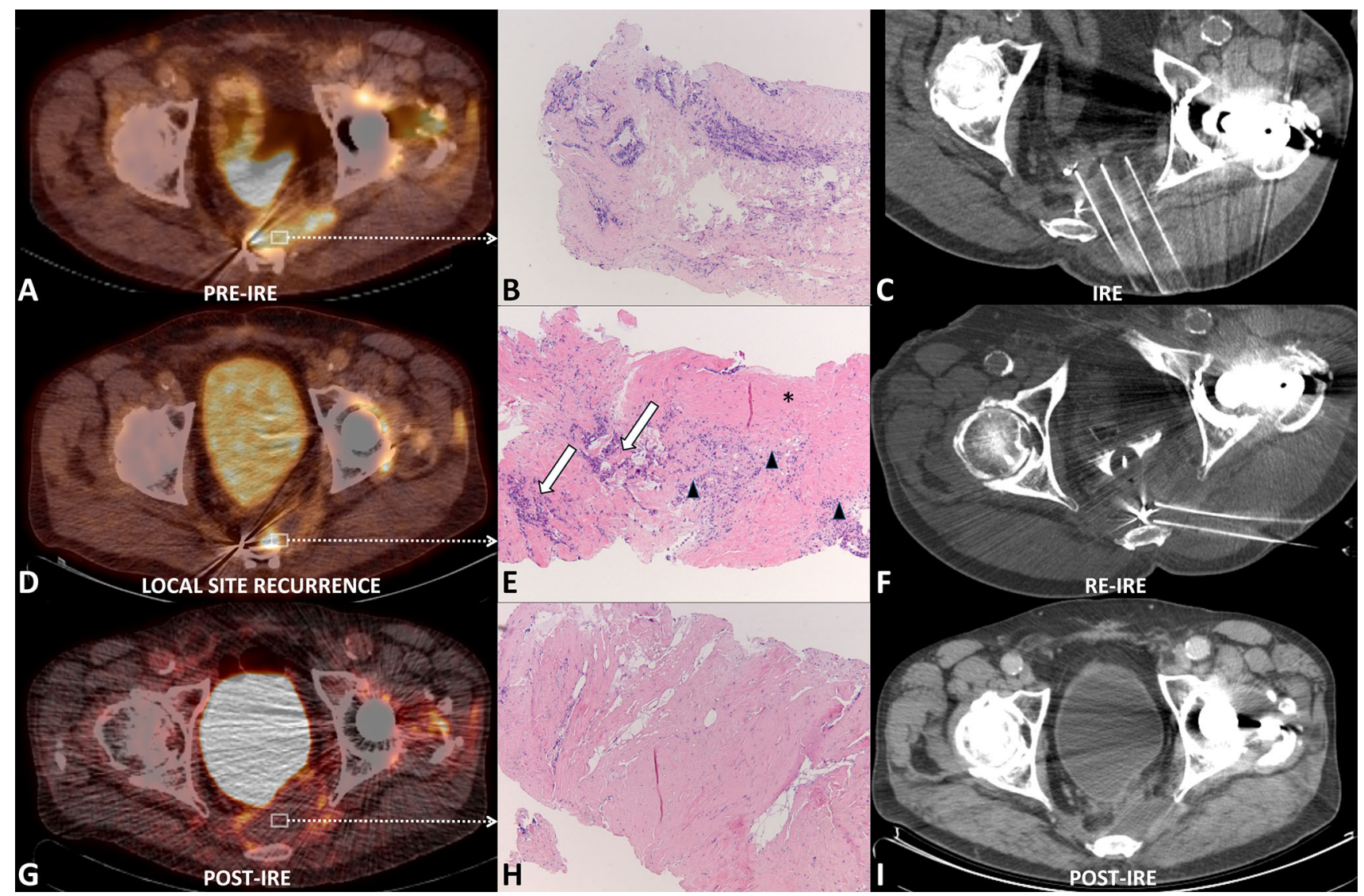

Fig. 2 18F-FDG PET-CT image (A) of a 70-year-old male patient with an 18F-FDG avid 60-mm pathologically proven locoregional recurrence (arrows) of primary rectal adenocarcinoma in the left parasacral area. Pre-IRE biopsy (B) of the initial LSR showing malignant cells on hematoxylin and eosin (HE) staining. Nonenhanced CT scan (C) showing three of the inserted needle electrodes prior to pulse delivery during the initial IRE procedure. 18F-FDG PET-CT image (D) 4 months after the initial IRE procedure showing a LSR. Pre-IRE biopsy (E) of the LSR prior to the second IRE procedure showing malignant cells (white arrows) encompassed by inflammatory cells (arrow heads); both embedded in fibrotic tissue (asterisks) on HE staining. Nonenhanced CT scan (F) showing two of the inserted needle electrodes just before pulse delivery during the second IRE procedure. 18F-FDG PET-CT image (G) 3 months after the second IRE procedure showing no signs for residual or recurring disease. Post-IRE biopsy $(\mathbf{H})$ of the ablated area after the second IRE procedure showing fibrotic tissue on HE staining. Nonenhanced CT scan (I) 6 months after the second IRE showing no signs of LSR

\section{Discussion}

Despite advances achieved in the radical treatment of primary colorectal and urogenital cancer, locoregional relapse remains a major therapeutic concern [1-4, 23]. This case series demonstrates the feasibility of IRE to achieve local tumor control (4/9 lesions, respectively, after one $[n=3]$ or two $[n=1]$ procedures) for heavily pre-treated pelvic tumor recurrences. Lesion size seems key in predicting outcome with only 1/4 LSR's for lesions $\leq 3 \mathrm{~cm}$. While safety should be confirmed in larger series, no major life-threatening complications occurred. Conversely, the number of patients suffering from permanent ablation-induced neural function loss should be considered high (6/8), which contradicts earlier animal studies $[14,16,17,24]$.
While tumor recurrences within the pelvis encompass a broad disease category, it is widely recognized that selected patients may benefit from intensive local therapy [25-27]. Surgical resection, often with curative intent, remains the treatment of first choice. However, the number of eligible patients is relatively small because the presence of adjacent crucial anatomical structures renders them unsuitable for complementary radical procedures [28]. Generally, the maximum tolerable radiation dose has already been reached during treatment of the primary tumor. In these cases, re-radiation is precluded due to the high risk of radiation-induced complications [4]. In the past two decades, thermal ablation has been investigated as treatment option for pelvic tumors. Apart from the (potentially) curative possibilities, thermal ablation can be valuable in the palliative setting to achieve cytoreduction 
and pain relief $[9,29]$. For example, palliative CT-guided RFA for painful pelvic recurrences of rectal cancer is considered a feasible and effective treatment in selected cases where the recurrence is located at a safe distance from major nerves, intestines, and urogenital tract structures [29-32]. In one study, twelve patients who underwent RFA for pain reduction were pain-free at the end of followup [29]. With one rectovesical fistula (8\%) and one rectal abscess (8\%), complication rate was considered acceptable. Since all lesions in our series were deemed unsuitable for thermal ablation, a fair comparison to these reports cannot be made. Yet, either RFA as IRE can induce the occurrence of post-procedural fistulas via the needle tracks. Up till now, only two case reports have been published regarding the use of IRE for the treatment of pelvic malignancies. In both cases, no major complications occurred [33, 34].

The actual working mechanism of IRE is based on a nonthermal effect; nonetheless, the development of some heat seems inevitable. Van den Bos et al. [35] found a temperature rise of $19.6{ }^{\circ} \mathrm{C}$ at $5 \mathrm{~mm}$ distance from the electrodes (using $1500 \mathrm{~V} / \mathrm{cm}$ ) in a nonperfused gelatine tissue model. According to animal studies, transient nerve dysfunction starts at temperatures as low as $40{ }^{\circ} \mathrm{C}$ [36] and permanent nerve injury at $51{ }^{\circ} \mathrm{C}$ [37]. Since the conductivity and the electric field distribution may differ between healthy and tumor tissue, this may have resulted in focal areas with a greater temperature rise, resulting in permanent thermal damage to the nervous structures [38]. The discrepancy between the preclinical animal experiments and our results regarding neural damage remains largely unclarified. One possible explanation is that the interelectrode distance in the animal experiments was smaller [approximately $5 \mathrm{~mm} ; 750 \mathrm{~V}(1500 \mathrm{~V} / \mathrm{cm})]$. Given the stronger impact on temperature development of adjusting voltage versus adjusting interelectrode distance [35], the temperature rise will be higher for settings used in clinical practice [approximately $20 \mathrm{~mm} ; 3000 \mathrm{~V}(1500 \mathrm{~V} / \mathrm{cm})]$. Another explanation can be electric field heterogeneity, as this is key in determining IRE outcome [39]. Although the parameters used in the animal studies were largely in accordance with ours, the induced potential depends on the ohmic characteristics of the tissue, which can differ in inhomogeneous tissues [40]. In the future, sequential pulsing, with pulse trains of 20 or 30 pulses, may prove to reduce the maximum tissue temperature gradient and therefore the extent and volume of thermal damage [35]. However, further research is needed to assess whether this is factual and whether this does not compromise oncologic efficacy. Furthermore, as opposed to healthy sciatic nerves and surrounding tissues in the animal experiments, the neural and perineural structures in the presented patients may well have been injured to some extent by earlier treatments or by tumor compression; by undergoing the
IRE procedure, they may have lost their neural function completely. Li et al. [14] explored the effect of IRE on nerves in a rat model and observed that nerve continuity was preserved post-procedurally. However, studies have shown that the regeneration process of nerves in larger animals is better comparable to neural regeneration in humans and might therefore be more suitable to study longer distance nerve regeneration [41, 42]. Schoellnast et al. $[16,17]$ assessed the acute, subacute, and delayed effects of IRE on the sciatic nerve in a pig model and concluded that IRE potentially damaged neural structures. They hypothesized that the observed preservation of the endoneurium architecture and the Schwann cell proliferation could potentially enable axonal regeneration. Most animals did not exhibit signs of lameness 1 month postIRE. Nevertheless, nerve conduction studies revealed residual neural function loss in half of the animals. These inconsistent results may be explained by the fact that the gluteal, extensor, and adductor muscles of the thigh can partially compensate for sciatic nerve palsy. In contrast to healthy individuals, cancer patients are generally less physically active and have less capacity to use other muscles to compensate the loss of neural function. In addition, preexisting pain complaints might hinder optimal recovery post-IRE. Recently, Tam et al. [43] investigated the post-ablation effects of IRE in the epidural space of the porcine spine and concluded that even at low electric field strengths nerve root injury can occur $(p \leq 0.05)$. To recognize impending nerve injury and to prevent irreversible damage, intraoperative neurophysiologic monitoring (IONM) might be helpful during percutaneous IRE, since neural structures adjacent to the target area are often poorly visible [44]. Chu et al. [45] confirmed that temporary axonal dysfunction can occur during IRE, and showed that IONM has the potential to detect conduction block at an early and reversible stage.

There were several inherent limitations in the present study. First, the study design was retrospective and the case number was limited. Second, the heterogeneity of tumor type and -size, anatomical location, and treatment indication (symptom palliation or disease control) was high. Another limitation was the inability to objectively quantify the level of nerve palsy after the ablation, especially given concomitant temporary muscle injury caused by IRE in some patients. To overcome this drawback in a future study, general neurological examination and nerve conduction studies could be performed to objectify the neural function pre- and post-IRE.

In conclusion, IRE may represent a suitable technique to treat well-selected locoregional tumors within the pelvis. However, as opposed to preclinical animal studies, permanent neural function loss can occur. Although complete ablation seems achievable for smaller lesions $(<3 \mathrm{~cm})$, this 
currently seems unrealistic for patients with larger tumors. For these patients, palliative care should be the primary focus.

\section{Compliance with Ethical Standards}

Conflict of interest M.R. Meijerink is paid consultant for AngioDynamics, and the other authors declare no conflict of interest.

Open Access This article is distributed under the terms of the Creative Commons Attribution 4.0 International License (http:// creativecommons.org/licenses/by/4.0/), which permits unrestricted use, distribution, and reproduction in any medium, provided you give appropriate credit to the original author(s) and the source, provide a link to the Creative Commons license, and indicate if changes were made.

\section{References}

1. Yeo HL, Paty PB. Management of recurrent rectal cancer: practical insights in planning and surgical intervention. J Surg Oncol. 2014;109(1):47-52.

2. Ang C, Bryant A, Barton DPJ, Pomel C, Naik R. Exenterative surgery for recurrent gynaecological malignancies. Cochrane Database Syst Rev. 2014;2:CD010449.

3. Colibaseanu DT, Dozois EJ, Mathis KL, et al. Extended sacropelvic resection for locally recurrent rectal cancer: can it be done safely and with good oncologic outcomes? Dis Colon Rectum. 2014;57(1):47-55.

4. Koom WS, Choi Y, Shim SJ, et al. Reirradiation to the pelvis for recurrent rectal cancer. J Surg Oncol. 2012;105(7):637-42.

5. Sole CV, Calvo FA, Lozano MA, et al. External-beam radiation therapy after surgical resection and intraoperative electron-beam radiation therapy for oligorecurrent gynecological cancer longterm outcome. Strahlenther Onkol. 2014;190(2):171-80.

6. Mohiuddin M, Marks G, Marks J. Long-term results of reirradiation for patients with recurrent rectal carcinoma. Cancer. 2002;95(5):1144-50.

7. Haie-Meder C, Morice P, Castiglione M. ESMO Guidelines Working Group. Cervical cancer: ESMO Clinical Practice Guidelines for diagnosis, treatment and follow-up. Ann Oncol. 2010;21(Suppl 5):v37-40.

8. Wiig JN, Søreide O. Locoregional recurrence of rectal cancer. In: Holzheimer RG, Mannick JA, editors. Surgical treatment evidence-based and problem-oriented. Munich: Zuckschwerdt; 2001. ISBN-10: 3-88603-714-2.

9. Mylona S, Karagiannis G, Patsoura S, Galani P, Pomoni M, Thanos L. Palliative treatment of rectal carcinoma recurrence using radiofrequency ablation. Cardiovasc Intervent Radiol. 2012;35(4):875-82.

10. Atwell TD, Carter RE, Schmit GD, et al. Complications following 573 percutaneous renal radiofrequency and cryoablation procedures. J Vasc Interv Radiol. 2012;23(1):48-54.

11. Phillips MA, Narayan R, Padath T, Rubinsky B. Irreversible electroporation on the small intestine. $\mathrm{Br} \mathrm{J}$ Cancer. 2012; 106(3):490-5.

12. Scheffer HJ, Nielsen K, de Jong MC, et al. Irreversible electroporation for nonthermal tumor ablation in the clinical setting: a systematic review of safety and efficacy. J Vasc Interv Radiol. 2014;25(7):997-1011.

13. Maor E, Ivorra A, Leor J, Rubinsky B. The effect of irreversible electroporation on blood vessels. Technol Cancer Res Treat. 2007;6(4):307-12.
14. Li W, Fan Q, Ji Z, Qiu X, Li Z. The effects of irreversible electroporation (IRE) on nerves. PLoS One. 2011;6(4):e18831.

15. Onik G, Mikus P, Rubinsky B. Irreversible electroporation: implications for prostate ablation. Technol Cancer Res Treat. 2007;6(4):295-300.

16. Schoellnast H, Monette S, Ezell PC, et al. Acute and subacute effects of irreversible electroporation on nerves: experimental study in a pig model. Radiology. 2011;260(2):421-7.

17. Schoellnast H, Monette S, Ezell PC, et al. The delayed effects of irreversible electroporation ablation on nerves. Eur Radiol. 2013; 23(2):375-80.

18. Seddon HJ. Three types of nerve injury. Brain 1943; 66(4):237-88.

19. Waldram M. Peripheral nerve injuries. Trauma 2003;5(2):79-96.

20. Eisenhauer EA, Therasse P, Bogaerts J, et al. New response evaluation criteria in solid tumours: revised RECIST guideline (version 1.1). Eur J Cancer. 2009;45(2):228-47.

21. Wahl RL, Jacene H, Kasamon Y, Lodge MA. From RECIST to PERCIST: evolving Considerations for PET response criteria in solid tumors. J Nucl Med. 2009;50(Suppl 1):122S-50S.

22. Goldberg SN, Grassi CJ, Cardella JF, et al. Image-guided tumor ablation: standardization of terminology and reporting criteria. Radiology. 2005;235(3):728-39.

23. Tam AL, Abdelsalam ME, Gagea M, et al. Irreversible electroporation of the lumbar vertebrae in a porcine model: is there clinical-pathologic evidence of neural toxicity? Radiology. 2014;272(3):709-19.

24. Wiggers T, Mannaerts GHH, Marinelli AWKS, Martijn H, Rutten HJT. Surgery for locally recurrent rectal cancer. Colorectal Dis. 2003;5(5):504-7.

25. Mannaerts GH, Martijn H, Crommelin MA, Dries W, Repelaer van Driel OJ, Rutten HJ. Feasibility and first results of multimodality treatment, combining EBRT, extensive surgery, and IOERT in locally advanced primary rectal cancer. Int J Radiat Oncol Biol Phys. 2000;47(2):425-33.

26. Gemignani ML, Alektiar KM, Leitao M, et al. Radical surgical resection and high-dose intraoperative radiation therapy (HDRIORT) in patients with recurrent gynecologic cancers. Int J Radiat Oncol. 2001;50(3):687-94.

27. Stelzer KJ, Koh W-J, Greer BE, et al. The use of intraoperative radiation therapy in radical salvage for recurrent cervical cancer: outcome and toxicity. Am J Obstet Gynecol. 1995;172(6):1881-8.

28. Friedlander M, Grogan M. Guidelines for the treatment of recurrent and metastatic cervical cancer. Oncologist 2002;7(4): $342-7$.

29. Pusceddu C, Sotgia B, Melis L, Fele RM, Meloni GB. Painful pelvic recurrence of rectal cancer: percutaneous radiofrequency ablation treatment. Abdom Imaging. 2013;38(6):1225-33.

30. Kalil AN, Santarosa F, Cunha L, et al. Radiofrequency ablation in the treatment of pelvic recurrence of rectal cancer. Hepatogastroenterology. 2003;50(54):1937-9.

31. Lefevre JH, Parc Y, Lewin M, Bennis M, Tiret E, Parc R. Radiofrequency ablation for recurrent pelvic cancer. Colorectal Dis. 2008;10(8):781-4.

32. Ohhigashi S, Watanabe F. Radiofrequency ablation is useful for selected cases of pelvic recurrence of rectal carcinoma. Tech Coloproctol. 2003;7(3):186-91.

33. Niessen C, Jung E-M, Schreyer AG, et al. Palliative treatment of presacral recurrence of endometrial cancer using irreversible electroporation: a case report. J Med Case Rep. 2013;7:128.

34. Kwok N, Lee LK, Arellano RS. Use of irreversible electroporation to treat metastatic pelvic lymphadenopathy. J Vasc Interv Radiol. 2016;27(8):1257-8.

35. Van den Bos W, Scheffer HJ, Vogel JA, et al. Thermal energy during irreversible electroporation and the influence of different ablation parameters. J Vasc Interv Radiol. 2016;27(3):433-43. 
36. Brodkey JS, Miyazaki Y, Erven FR, Mark VH. Reversible heat lesions with radiofrequency current. a method of steriotactic localization. J Neurosurg. 1964;21:49-53.

37. Bunch TJ, Bruce GK, Mahapatra S, et al. Mechanisms of phrenic nerve injury during radiofrequency ablation at the pulmonary vein orifice. J Cardiovasc Electrophysiol. 2005;16(12):1318-25.

38. Nickfarjam A, Firoozabadi SMP. Parametric study of irreversible electroporation with different needle electrodes: electrical and thermal analysis. Int J Hyperthermia. 2014;30(5):335-47.

39. Miller L, Leor J, Rubinsky B. Cancer cells ablation with irreversible electroporation. Technol Cancer Res Treat. 2005;4(6): 699-705.

40. Bertacchini C, Margotti PM, Bergamini E, Lodi A, Ronchetti M, Cadossi R. Design of an irreversible electroporation system for clinical use. Technol Cancer Res Treat. 2007;6(4):313-20.

41. Fullarton AC, Lenihan DV, Myles LM, Glasby MA. Obstetric brachial plexus palsy: a large animal model for traction injury and its repair. Part 1: age of the recipient. J Hand Surg Br. 2000;25(1):52-7.

42. Hess JR, Brenner MJ, Fox IK, et al. Use of cold-preserved allografts seeded with autologous schwann cells in the treatment of a long-gap peripheral nerve injury. Plast Reconstr Surg. 2007;119(1):246-59.

43. Tam AL, Figueira TA, Gagea M, et al. Irreversible Electroporation in the Epidural Space of the Porcine Spine: Effects on Adjacent Structures. Radiology. 2016;281(3):763-771.

44. Marshall RH, Avila EK, Solomon SB, Erinjeri JP, Maybody M. Feasibility of intraoperative nerve monitoring in preventing thermal damage to the "Nerve at Risk" during image-guided ablation of tumors. Cardiovasc Intervent Radiol. 2016;39(6): 875-84.

45. Chu J, Nagata M, Chen $X$, et al. Irreversible electroporationinduced sciatic neuropathy observed by intraoperative neuromonitoring. Clin Neurophysiol. 2016;127(8):2770-2. 